\title{
Modern quantitative genetics: Dissecting complex polygenic systems into individual genetic factors
}

Modern quantitative genetics began in the late 1980s with the advent and application of molecular marker technology. Molecular markers made it possible to genotype the entire genome of an individual. Thus, for the first time, quantitative genetic analysis could be performed based on phenotypic and genotypic information. As a result, quantitative genetic studies were no longer limited to the estimation of genetic parameters by treating the polygenic system as a whole, but were able to identify the locations and effects of individual quantitative trait loci (QTLs) as well as to examine the interactions between QTLs. Since the early 1990s, molecular marker technology has developed very rapidly. In particular, during the last decade, high-throughput genomic approaches such as microarray and next generation sequencing have been developed, making possible the simultaneous detection of tens or hundreds of thousands of polymorphic loci. These approaches have greatly enhanced the efficiency of molecular marker analysis, making genome genotyping easier and no longer a bottleneck in genetic studies. The fast progress of molecular marker technology has significantly advanced the development of modern quantitative genetics, making it one of the hottest research areas in life sciences.

In this special issue on quantitative genetics, fourteen articles contributed by Chinese scientists are presented. The articles cover a wide range of modern quantitative genetics such as QTL mapping methodology, computational tools for quantitative genetic analysis, experimental researches in QTL mapping, and marker-assisted breeding. The first article [1] is a review of statistical approaches for QTL mapping and marker-assisted breeding that were developed by the authors and their group. In subsequent articles, several new QTL mapping methods are proposed for specific experimental designs, including (1) the use of two backcross populations [2], (2) the use of a NCIII design based on recombinant inbred lines [3], and (3) the use of chromosome segment substitution lines [4]; or for specific purposes, namely, (1) to detect epistasis between nuclear genes (QTLs) and the cytoplasm [5], and (2) to map sterile genes (QTLs) with epistasis in remote hybridization of plant [6]. In another article [7], an improved approach for QTL mapping in farm animals based on general pedigrees, termed pedigree transmission disequilibrium test, is proposed; this method performs better than existing statistical methods.

While marker-dependent QTL mapping remains the primary method used in modern quantitative genetic researches, a new and different idea is proposed in the cover article [8]. The aim of this approach is to connect quantitative traits with genes without the need of molecular markers. The authors describe a two-step statistical approach, first, to detect the association of phenotypic variation in a diallel cross experiment between a quantitative trait and gene expression profiles measured by microarrays, and then to estimate the contribution of individual genes to the genetic variation of the target trait. This approach is interesting and promising because it can potentially be used to uncover the underlying genes of the target traits straightforwardly, thereby providing a novel way to dissect the complex genetic basis of quantitative traits.

Apart from the QTL mapping methods, other related statistical issues are also addressed. One of the articles [9] demonstrates how to evaluate the efficiency of different QTL mapping methods by estimating their statistical power and false discovery rate through computer simulation. Another article [10] proposes a statistical model for estimating and testing the linkage between molecular markers in a triploid population. This model may serve as a theoretical basis for the genetic study of quantitative variation in polyploid species. In one more article [11], a software package for quantitative genetic analysis is presented. The software can be used to handle a wide range of experimental data from different sources, such as diallel crosses, regional trials, core germplasm collections, QTL mapping, and microarrays. Finally, three of the articles report practical studies using modern quantitative genetic theory and methodology, including QTL mapping for anxiety-related traits in mice [12], association analysis between growth traits and candidate genes involved in crucial metabolism pathways in pigs [13], and marker-assisted genome-wide selection for genetic improvement of two important traits in beef cattle [14].

In summary, modern quantitative genetics is an important research area that attracts broad interest among researchers in life sciences. Its main goal is to illuminate the polygenic basis of quantitative traits so as to guide the genetic improvement of plants and animals and the therapy of many complex human diseases. This special topic provides the reader with a "banquet" 
of new achievements in modern quantitative genetics, with fourteen delicious "dishes" of different flavors "cooked" by our Chinese colleagues. I hope that it will be an enjoyable feast.

1 Xu H M, Zhu J. Statistical approaches in QTL mapping and molecular breeding for complex traits. Chin Sci Bull, 2012, 57: 2637-2644

2 Zhu Z H, Hayart Y, Yang J, et al. Statistical method for mapping QTLs for complex traits based on two backcross populations. Chin Sci Bull, 2012, 57: 2645-2654

3 He X H, Hu Z L, Zhang Y M. Genome-wide mapping of QTL associated with heterosis in the RIL-based NCIII design. Chin Sci Bull, 2012, 57: 2655-2665

4 Tang Z X, Xiao J, Hu W M, et al. Bin-based model construction and analytic strategies for dissecting complex traits with chromosome segment substitution lines. Chin Sci Bull, 2012, 57: 2666-2674

5 Tang Z X, Hu Z Q, Yang Z F, et al. Framework for dissection of complex cytonuclear epistasis by two-dimensional genome scan. Chin Sci Bull, 2012, 57: 2675-2680

6 Xie S Q, Chen J G. A statistical method for the genetic mapping of sterile genes that exhibit epistasis in remote hybridization of plant using molecular markers in an $\mathrm{F}_{2}$ population. Chin Sci Bull, 2012, 57: 2681-2687

7 Ding X D, Wang C L, Zhang Q. Pedigree transmission disequilibrium test for quantitative traits in farm animals. Chin Sci Bull, 2012, 57: 2688-2694

8 Yang D G, Ye C Y, Ma X F, et al. A new approach to dissecting complex traits by combining quantitative trait transcript (QTT) mapping and diallel-cross analysis. Chin Sci Bull, 2012, 57: 2695-2700

9 Li H H, Zhang L Y, Wang J K. Estimation of statistical power and false discovery rate of QTL mapping methods through computer simulation. Chin Sci Bull, 2012, 57: 2701-2710

10 Wang J X, Dong X L, Pang X M, et al. Simultaneous estimation of genetic linkage and preferential pairing factor for a triploid population with unphased markers. Chin Sci Bull, 2012, 57: 2711-2720

11 Chen G B, Zhu Z X, Zhang F T, et al. Quantitative genetic analysis station for the genetic analysis of complex traits. Chin Sci Bull, 2012, 57: 2721-2726

12 Zhu Z H, Zhang C H, Wang X S, et al. Dissecting anxiety-related QTLs in mice by univariate and multivariate mapping. Chin Sci Bull, 2012, 57: 2727-2732

13 Yang F, Wang Q P, Wang M H, et al. Associations between gene polymorphisms in two crucial metabolic pathways and growth traits in pigs. Chin Sci Bull, 2012, 57: 2733-2740

14 Zeng Z Y, Tang G Q, Ma J D, et al. Developing a genome-wide selection model for genetic improvement of residual feed intake and carcass merit in a beef cattle breeding program. Chin Sci Bull, 2012, 57: 2741-2746

WU WeiRen

College of Life Sciences, Fujian Agriculture and Forestry University, Fuzhou 350002, China

Open Access This article is distributed under the terms of the Creative Commons Attribution License which permits any use, distribution, and reproduction in any medium, provided the original author(s) and source are credited. 